\title{
Testing of Large Optical Surfaces With Small Test Plates
}

\author{
James B. Saunders
}

\begin{abstract}
A procedure for testing large optical surfaces with relatively small optical standards is described. Simplified formulas are used to apply a statistical method for obtaining increased precision. A practical example is used to illustrate the procedure for testing surfaces that may be assumed to have revolution symmetry.
\end{abstract}

\section{Introduction}

With the increased use of massive optical parts, there is need for a practical method of testing large optical surfaces with relatively small standards, such as are available in the average optical shop. The conventional test of observing fringes between the standard and unknown at various positions on the surface enables the optician to form a rough estimate of the nature of the surface. For more orecise values either extensions of the present conventional tests or new methods must be used. Present shop methods of testing optical surfaces are not practical for massive optical elements as standard test plates of the sizes required for conventional test methods are seldom available.

A test for shape of very large optical surfaces, by means of interference of light, may be made with a standard test plate (flat or spherical) that is much smaller than the surface to be tested. The principles involved are not new and may be considered elementary, but those who perform the tests on optical surfaces during the polishing are not as a rule sufficiently versed in mathematics to apply the principles outlined in the present paper to the best advantage. They can, however, apply observed data to a set of simple formulas and compute the ensuing results.

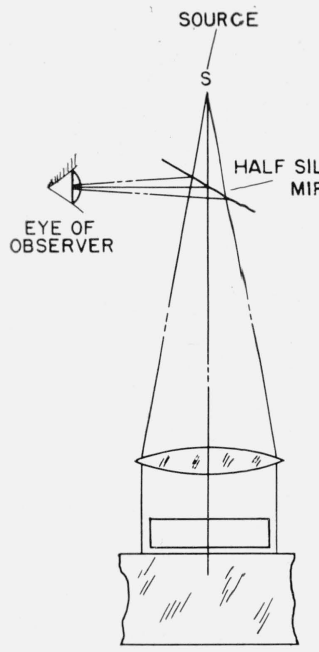

A

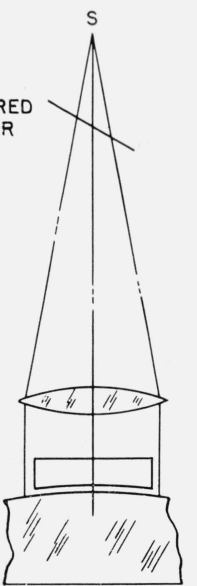

B

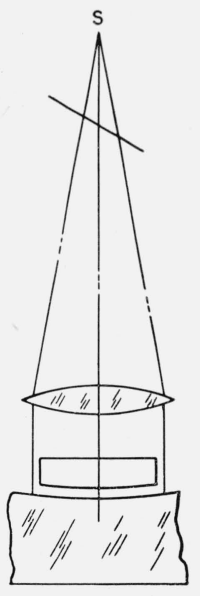

C
Figure 1. Optical arrangements for testing (A) flat surfaces, $(B)$ convex surfaces, and $(C)$ concave surfaces.
A procedure is described in which an ordinarysized standard test plate can be used to measure the shape of surfaces that are much larger than the standard. Use is made of statistical methods for improving the computed results. Simplified formulas will be developed that may be applied by opticians, after a short training period, even though they may not be able to follow the mathematical derivations and theory. The application of the general formulas to a typical set of data will then be made to illustrate the testing of a surface by means of a smaller standard optical flat. The method is not limited to plane surfaces. The sphericity of very large spherical or aspherical surfaces may be measured with standard test plates of approximately the same radius, if the viewing system permits the use of normal incident light. Figure 1, (A, B, C) shows simple optical arrangements for testing plane, convex, and concave surfaces, respectively.

The basic principle may also be applied to the testing of off-axis curved, or nonsymmetrical, surfaces, with modifications of the formulas. However, as these are encountered only rarely, the present paper will be limited to surfaces of revolution.

We will consider a Fizeau viewer (iig. 1) that has an aperture equal to or greater than the area of the standard surface. Spherical surfaces are referred to the spherical master with which they are tested, and plane or approximately plane surfaces are referred to planes. The equations and measurements are identical for plane and spherical surfaces. This discussion will deal with the testing of an approximately plane surface against a standard optical flat. The unknown surface is assumed to be a figure of revolution about an axis normal to and passing through a known point on it. In general, this point will be at or near the center, if the surface is circular.

\section{Experimental Procedures}

If the standard flat is adjusted normally to the collimated beam of light and the unknown is placed close to and approximately parallel to the standard, interference fringes may be observed with mono chromatic light of known wavelength. Measurements made on the fringe pattern permit a computation of the shape of the unknown relative to a plane (sphere for spherical surfaces) over the visible region of interference. If adjustments permitted, the entire area of the unknown could be covered by 
moving the standard step by step without rotation, in a plane parallel to the standard plane. The aggregate of the resultant fringe patterns, when properly assembled to form a composite pattern of the whole surface, would be quite similar to what would have been obtained with a standard surface that covered the whole of the unknown.

As it is virtually impossible to make the above assumed adjustments, corrections for rotation and changes in separation of the unknown, relative to the original surface of the standard, must be made by measuring these changes and applying the required corrections. The corrections are always subject to errors of observation. If several successive positionings of the standard, relative to the unknown, are required to measure the shape along a diameter, these errors accumulate. Consequently, it is desirable to reduce these errors. The "Method of Averages," 1 which is easy to apply and yields simple working formulas, is used since it provides adequate precision.

In general, large optical surfaces are ground and polished by machines that produce figures of revolution about a known point, usually located at the center of the surface. If the surface is one of revolution, ${ }^{2}$ its departure from a straight line that is tangent to it at the center of revolution is a measure of its departure from its tangent plane and consequently determines the shape of the entire surface relative to any other chosen plane. The axis of abscissas (see fig. 2) is chosen as the intersection of a plane through the axis of rotation of the surface with the plane that is tangent to the surface at its center. The unit of abscissas is chosen as the separation of equally spaced reference marks along a chosen diameter of the surface. The chosen diameter is the axis of abscissas. The axis of ordinates is the axis of revolution of the surface. The unit of ordinates is one-half the wavelength of the light used.

The following steps are the chosen procedure for acquiring the data necessary to compute the shape of the surface. The master flat is placed on, and concentric with, the surface (positions $A$ and $A^{\prime}$, fig. 2). The magnitude of the air wedge between the two surfaces is adjusted to produce a satisfactory number of fringes in the field (see fig. 3). The direction of this wedge is adjusted to make the fringes approximately normal to the line along which the chosen reference points lie. Except for plane surfaces this wedge varies from one reference point to the next. Consequently, if linear interpolation is used in estimating fractions of fringes at the reference points, the error in estimation varies with the fraction observed. For most observers this error is a minimum when the fraction is 0.0 or 0.5 , that is, when the reference point falls on the center of a dark or a bright fringe. Best accuracy is obtained by reading abscissas corresponding to the center of all fringes - both dark and bright - and from these data, by nonlinear interpolation, compute the relative

\footnotetext{
1 J. B. Scarborough, Numerical mathematical analysis, p. 446 (Johns Hopkins Press, Baltimore, Md., 1950).

2 W. A. Granville, Elements of differential and integral calculus, p. 264 (Ginn \& Co., New York, N. Y., 1911).
}

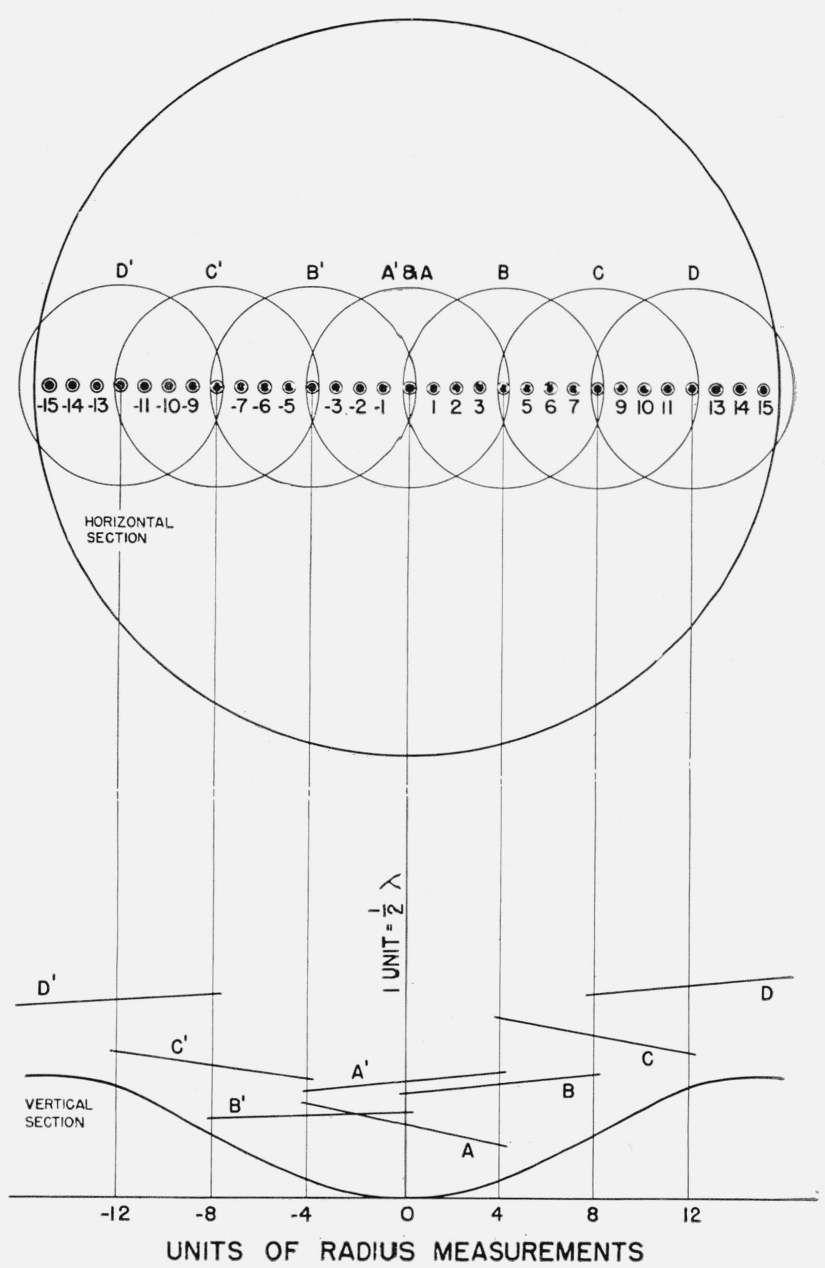

Figure 2. Coordinate system of reference.

The vertical section (below) cuts the horizontal section (above) along the line of reference points - dots concentric with small circles. Circles A, A' B, B', ete. represent the several positions of the standard test plate. The corresponding straight lines below represent sections through the standard surface of reference.

orders at the chosen reference points. The procedure will be explained in greater detail with the held of the fringe configuration shown in figure 3.

The fringes (relative orders of interference) in position $A$ are evaluated at the several equally spaced reference points by the method indicated above. The standard is then moved to position $B$, which overlaps an appropriate amount of the area covered in its first position, $A$. Again the fringe readings at all reference points, covered by the standard in this new position, are evaluated. The standard is then moved to position $C$ and the corresponding fringe readings noted. This procedure is repeated until the surface covered by the master extends to the edge of the unknown surface of revolution. The positions represented by primed letters are a second independent set, useful in checking the precision of the method.

In general, the fringe values at the reference points will not be integral. Consequently, the fractional parts must be obtained by interpolation and, in marginal cases, by extrapolation. Linear interpolation 


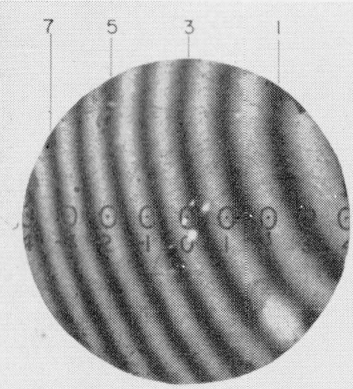

A

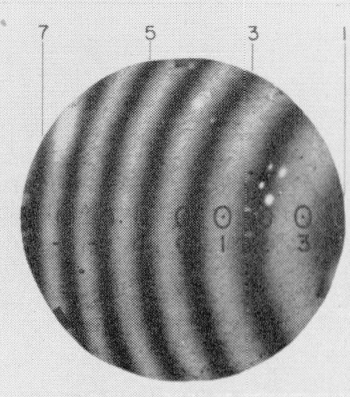

$A^{\prime}$

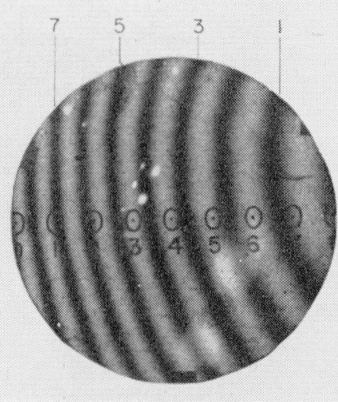

B

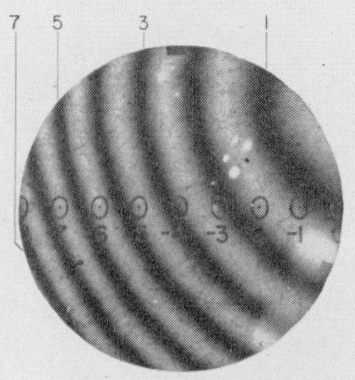

$B^{\prime}$

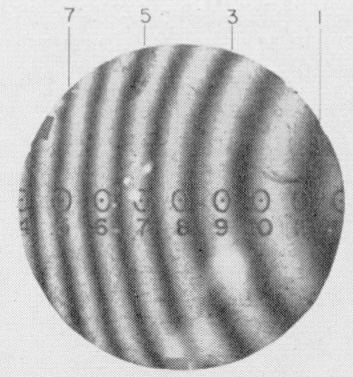

C

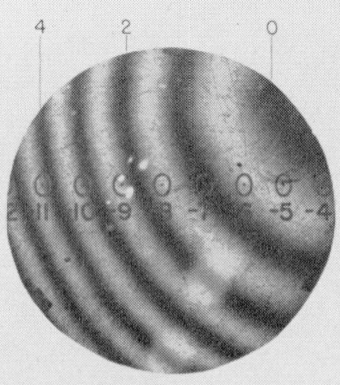

$C^{\prime}$

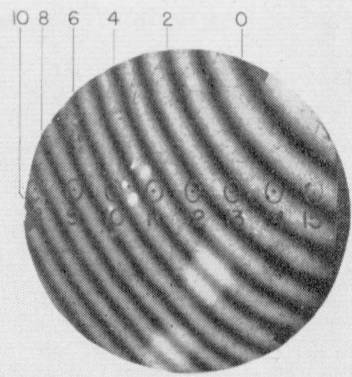

D

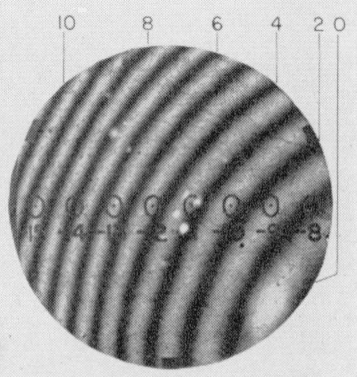

D'

Figure 3. Fringes for the several positions of the standard test plate.

The numbers above each picture represent relative orders of interference.

or extrapolation is accurate only when the fringes are equally spaced along the straight line on which the reference points are chosen. If they are equally spaced, the surface is plane and no further test is necessary. In general, the fringes will be curved and nonlinear interpolation should be used if highest accuracy is to be obtained. Of the several methods of performing nonlinear interpolation, the graphical method is simplest and, for these purposes, adequate. The details of this are best shown by a description of its application to actual data. This will be given in a later section, where the shape of the surface of a glass disk will be computed from photographs of interference fringes that are obtained when the glass disk is tested interferometrically against a standard flat.

\section{Derivation of Formulas}

A set of formulas are now derived for use in the above-mentioned computation. In figure 4 the curved line represents the surface to be measured. The chosen reference plane is represented by the axis of abscissas. The chosen reference points are indicated by circles on the curved line. The several positions of the master plane relative to the coordinate system are represented by straight lines at various angles. The coordinates of all chosen reference points, which are marked on the surface of the unknown, are $\left(R, Y_{R}\right)$. The observed fringe (a relative order of interference) at $\left(R, Y_{R}\right)$ is designated
$A_{R}$ for the first position of the standard, $B_{R}$ for the second position of the standard, $C_{R}$ for the third position, and so forth. The slopes and intersections of the straight lines with the axis of ordinates are represented by $M_{A}$ and $A_{0}$, respectively, for the $A$ position, $M_{B}$ and $B_{0}$ for the $B$ position, $M_{C}$ and $C_{0}$ for the $C$ position, and so forth.

The slope-intercept form of the equation for the straight line representing the standard in the $X$ position $(X=A, B, C$, etc. $)$ is

$$
Y_{R}+\left(X_{R}-X_{0}\right)=M_{X} R \text {. }
$$

In position $A$, for example, the ordinates of the line are $\left(A_{R}+Y_{R}\right)=A_{0}+M_{A} R$, where $A_{R}$ is the separation of the two optical surfaces. The absolute values for $X_{R}$ are unknown, but differences in $X_{R}$, for any given position of the standard, are directly observable. Consequently, the integral part of the smallest $X_{R}$ will be subtracted from all $X_{R}$ 's for purpose of computation. The quantity $\left(X_{R}-X_{0}\right)$ is unaffected by this operation.

We will evaluate $M_{X}$ and $X_{0}$ by applying the method of averages (footnote 1) to the data. The grouping of observations will be made in such manner that there is no overlapping of the two groups of points except when an odd number of reference points are to be applied to an evaluation. When the number of reference points in the two groups is odd, two observations are considered to be made at each of the points, and the two observations made at the central 


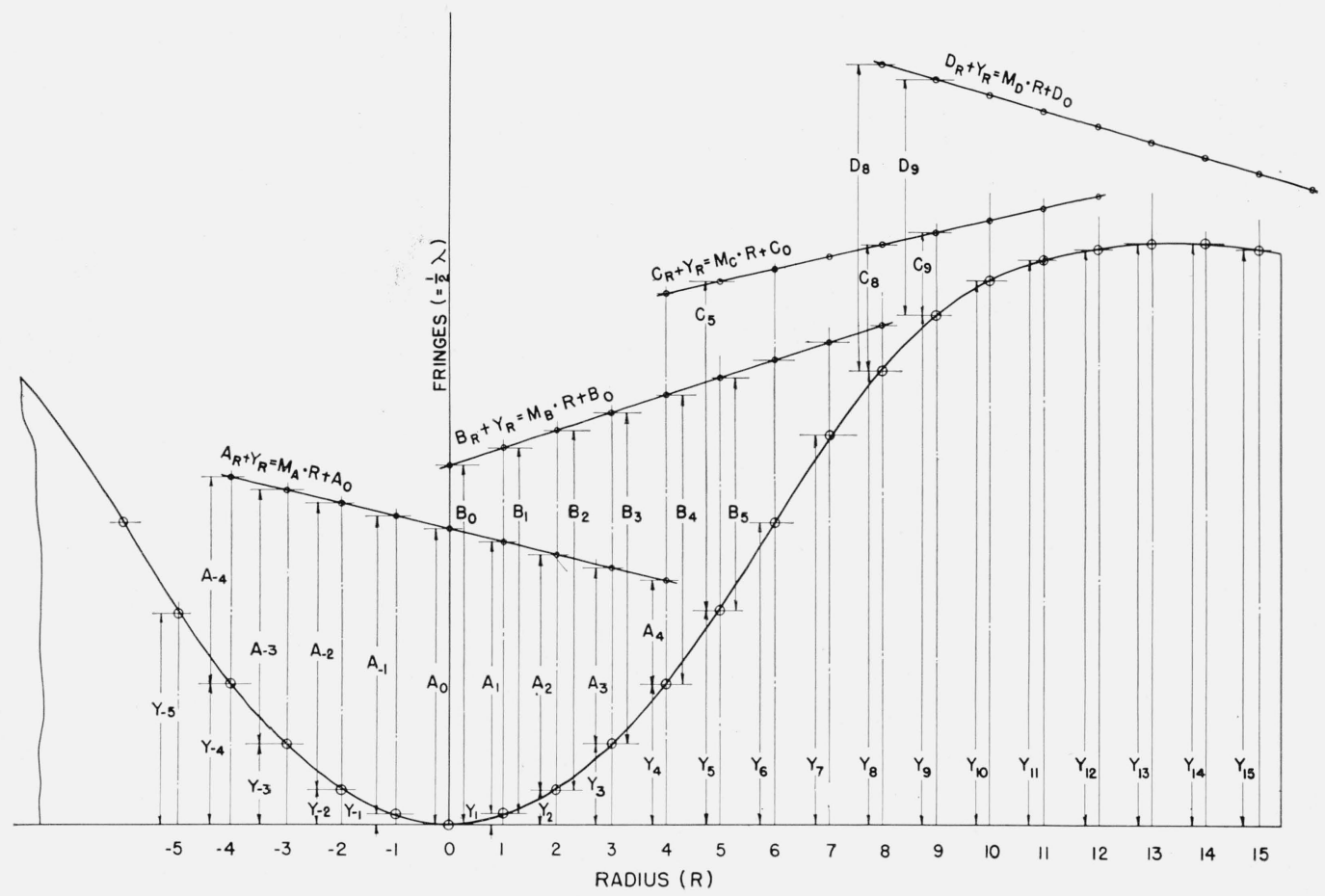

Figure 4. Analytical representation of the surface and standard for mathematical analysis.

The slanting straight lines represent the several positions of the standard and the adjacent formulas the equations of its corresponding locii.

point are divided between the two groups. The two groups then have an equal number of observations, and all observations are thereby assigned equal weights. When the total number of reference points is even, there is no overlapping of groups for an equal number of points to be allocated to each of the two groups for equal weighting of datum.

In applying the method of averages (footnote 1), we require that the algebraic sum of errors be zero for each of the two groups of abservations. This requirement is represented by the following equations:

$$
\left.\begin{array}{l}
\sum_{R_{1}}^{R_{2}}\left(Y_{R}+X_{R}\right)=M_{X} \sum_{R_{1}}^{R_{2}} R+\frac{1}{2} N_{X} X_{0}, \\
\sum_{R_{3}}^{R_{4}}\left(Y_{R}+X_{R}\right)=M_{X} \sum_{R_{3}}^{R_{4}} R+\frac{1}{2} N_{X} X_{0},
\end{array}\right\}
$$

where summations in the first of this pair of equations are from the lowest value of $R\left(=R_{1}\right)$ to the largest value of $R\left(=R_{2}\right)$ in this group and summations in the second equation are from the lowest value of $R\left(=R_{3}\right)$ in the second group to the largest value of $R\left(=R_{4}\right)$ in the second group. Equality of $R_{2}$ and $R_{3}$ will result when the number of reference points is odd, whereas they will differ by unity when the total number of reference points is even. The number of reference points used in each evaluation is $N_{X}$.

Solving for $M_{X}$ and $X_{0}$ from the pair of equations (2), we get

$$
M_{X}=\frac{\sum_{R_{3}}^{R_{4}}\left(Y_{R}+X_{R}\right)-\sum_{R_{1}}^{R_{2}}\left(Y_{R}+X_{R}\right)}{\sum_{R_{3}}^{R_{4}} R-\sum_{R_{1}}^{R_{2}} R}
$$

$$
X_{0}=\frac{2\left[\sum_{R_{3}}^{R_{4}} R \sum_{R_{1}}^{R_{2}}\left(Y_{R}+X_{R}\right)-\sum_{R_{1}}^{R_{2}} R \sum_{R_{3}}^{R_{4}}\left(Y_{R}+X_{R}\right)\right]}{N_{X}\left[\sum_{R_{3}}^{R_{4}} R-\sum_{R_{1}}^{R_{2}} R\right]}
$$

Equations (1), (3), and (4), together with the assumption that the surface to be measured has revolution symmetry, permit a computation of $Y_{R}$ for all chosen reference points and consequently the shape of the surface.

The foregoing analysis permits a statistical evaluation of $M_{X}$ and $X_{0}$ for all values of $X$ (i. e., $A, B$, $C, \ldots$. except the quantity $A_{0}$. An error in $A_{0}$ represents an error in the position of the reference plane and since this has no effect on the computed shape of the surface, the observed $A_{0}$ is assumed to be free from error.

As the surface is assumed to have revolution symmetry,

$$
Y_{R}=Y_{-R} .
$$

From eq (1) $Y_{R}=M_{A} R+\left(A_{0}-A_{R}\right)$, and $Y_{-R}=$ $-M_{A} R+\left(A_{0}-A_{-R}\right)$. On eliminating $M_{A}$ and $Y_{R}$ from these three equations, we have

$$
Y_{R}=A_{0}-\frac{1}{2}\left(A_{R}+A_{-R}\right) .
$$

As $M_{A}$ does not enter into the computation, it need not be evaluated.

\section{Illustrative Example}

Equations (1), (3), (4), and (6) form a set of fundamental equations from which all values for $Y_{R}$ may be computed. 
To clarify any possible lack of understanding in the development and application of these formulas, we will apply them to a set of observations and will compute the resultant shape of a surface. In this case the large circle in figure 2 represents the periphery of a 31.1-cm-diameter disk that is to be measured by means of a standard whose diameter is $8.2 \mathrm{~cm}$. The seven circles, centered on one diameter of the large disk, represent the several successive positions of the standard. The eight circular fringe patterns shown in figure 3 (forming two independent sets, primed and unprimed) were obtained by photographing the fringe patterns formed by light reflected normally from the top surface of the large disk and the standard flat when in the several positions indicated in figure 2. The differences in values of the ordinates $\left(Y_{R}-Y_{-R}\right)$, obtained from the two sets of photographs, is a measure of the accuracy obtainable. These differences are due to errors of observation and to an error in the choice of the center point. If the unknown is ground and polished on a spindle that is not concentric with it, the axis of rotation of the unknown surface will not be centered at the chosen origin of coordinates. However, the average value of $Y_{R}$ and $Y_{-R}$ from the two sets of data, primed and unprimed, respectively, will be almost free from the error of centering.

In order to obtain more precise values for $X_{R}$, the following procedure is followed: Reference marks with their associated $R$-values, or abscissas, are placed on the glass surface along the chosen reference line. These reference points are indicated by black dots centered in the small circles shown in figure 2. The standard is placed in the desired position and the air wedge adjusted so that a desirable number ( 5 to 10) of fringes cross the reference line. The present author prefers to have the fringes approximately perpendicular to the reference line. Considerable departure from this, however, is usually tolerated. The fringe pattern is photographed. ${ }^{3}$ This is repeated for all desired positions of the standard. A fine straight line, using a needle point and a straight edge, is drawn across the photograph through the nine reference points on it. The positions of the centers of the references points are marked by pricking the photograph with a needle. This is to permit accurate readings on their positions. If no distortion is introduced in the photographic reproduction, the separation of all adjacent points will be equal. The intersections of all fringes, both dark (i. e., integral orders of interference) and light (integral plus half orders), with the reference line are marked also by pricking the photograph. The direction of increasing orders of interference is ascertained from tests and notations made when the fringes are photographed. The relative orders of interference, beginning with 1 , are indicated by inserting numbers on the dark fringes. In figure 3 these numbers appear above each of the fringe patterns. The lesser visible numbers, adjacent to the reference points, represent the abscissas (or

${ }^{3}$ A micrometer eyepiece may be used to read the data directly and thus dispense with photography.
$R$-values) of the several reference points that were marked on the unknown surface.

In general, the scale of the photographic reproduction will differ from that of the chosen abscissa scale. For interpolation purposes any convenient scale may be used to measure the relative positions of points on the photograph. A centimeter scale with millimeter marks was used for this compilation. We will define the scale used as the $P$-scale and the readings from it as $P$-values. The $P$-values corresponding to all reference points and fringes are read off the centimeter scale and inserted in column 1 of table 1, which represents a typical data sheet. The corresponding $R$-values (numbers associated with the fiduciary reference points and appearing in the photographs) are inserted in column 2 ; the $A_{R^{-v}}$ alues, corresponding to integral and half-integral orders of interference, are inserted in their respective places in column 3 . If an observed $P$-value, corresponding to an $R$-value, falls precisely on a fringe (reference point centered on a fringe), two identical $P$-values are inserted as for $R=-2$ and $A_{R}=5.5$ in the case given here.

The object of this listing and the following computations is to obtain more precise $A_{R^{-v}}$ alues (orders of interference) than can be obtained by direct interpolation or reading from the photographs.

A direct plot of the $P$-values versus $A_{R^{-v}}$ alues first entered in column 3 , table 1 (the blank spaces to be filled later), shown as small black dots in figure 5, may be made. A smooth curve is then drawn through these points. The values for $A_{R}$ in column 3 , which correspond to $R$-values in column 2 , are located on the curve. The corresponding ordinates represent the interpolated $A_{R^{-} \text {values desired. The }}$ values with asterisks, shown in table 1, are more precise values obtained by an interpolation process described in the following paragraphs.

The values for $A_{R}$, determined above from the direct plot, are not as precise as the directly observed values from which the plot was made. More precise

\section{TABle 1. Arrangement of data for computation}

Values for $A_{R}$ in column 3 that are followed by an asterisk are interpolated values from the curve of figure 5 .

\begin{tabular}{|c|c|c|c|c|}
\hline$P$ & $R$ & $A_{R}$ & $0.7 P$ & $\left(0.7 P+A_{R}\right)$ \\
\hline $\begin{array}{r}0.21 \\
.44 \\
.93 \\
1.39 \\
1.54 \\
1.85 \\
2.40 \\
2.94 \\
2.94 \\
3.54 \\
4.20 \\
4.29 \\
4.80 \\
5.43 \\
5.64 \\
6.05 \\
6.85 \\
7.00 \\
7.70 \\
8.37 \\
8.60 \\
9.67 \\
9.73 \\
10.80 \\
11.08\end{array}$ & $\begin{array}{c}-4 \\
-3 \\
-2 \\
-1 \\
0 \\
1 \\
2 \\
3 \\
4\end{array}$ & $\begin{array}{l}8.23^{*} \\
8 \\
7.5 \\
7 \\
6.80^{*} \\
6.5 \\
6 \\
5.50^{*} \\
5.5 \\
5 \\
4.5 \\
4.42^{*} \\
4 \\
3.5 \\
3.33^{*} \\
3 \\
2.5 \\
2.41^{*} \\
2 \\
1.62^{*} \\
1.5 \\
1 \\
0.98^{*} \\
.5 \\
.39^{*}\end{array}$ & $\begin{array}{l}0.15 \\
.31 \\
.65 \\
.97 \\
1.08 \\
1.29 \\
1.68 \\
2.06 \\
2.06 \\
2.48 \\
2.94 \\
3.00 \\
3.36 \\
3.80 \\
3.95 \\
4.24 \\
4.80 \\
4.90 \\
5.39 \\
5.86 \\
6.02 \\
6.77 \\
6.81 \\
7.56 \\
7.76\end{array}$ & $\begin{array}{l}8.38^{*} \\
8.31 \\
8.15 \\
7.97 \\
7.88^{*} \\
7.79 \\
7.68 \\
7.56^{*} \\
7.56 \\
7.48 \\
7.44 \\
7.42^{*} \\
7.36 \\
7.30 \\
7.28^{*} \\
7.24 \\
7.30 \\
7.31^{*} \\
7.39 \\
7.48^{*} \\
7.52 \\
7.77 \\
7.79^{*} \\
8.06 \\
8.15^{*}\end{array}$ \\
\hline
\end{tabular}




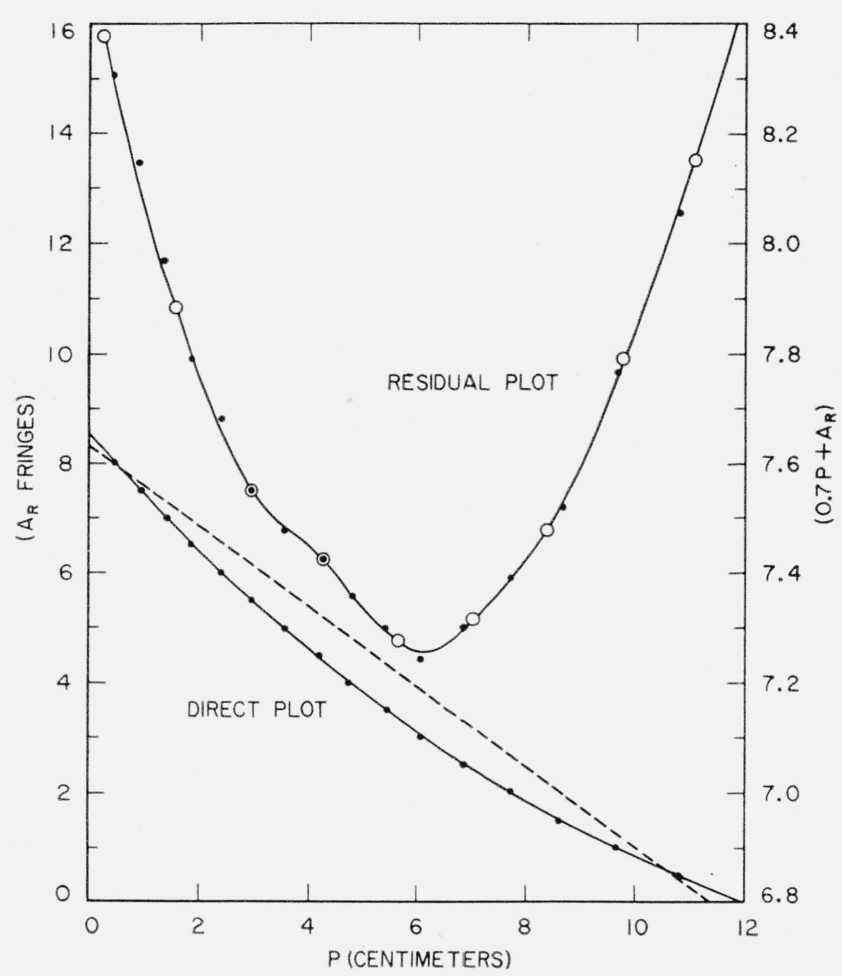

FIGURE 5. Direct and residual plot of data for nonlinear interpolation of fringe orders.

values may be obtained from a residual plot. ${ }^{4}$ To obtain data for the residual plot, a column of values (table 1, column 4), computed with a slide rule, is added to the table of observed quantities. This column is a product of corresponding $P$-values and an appropriately chosen constant. This constant represents the slope of a straight line that crudely approximates the plotted points. It is indicated in figure 5. The approximate slope of this line is 0.7 . A relatively large error in the choice of the slope contributes little or no error in the final results. Values of $0.7 P$ are accordingly computed to two decimal places. The sums of corresponding values from columns 3 and 4 , except where vacancies exist in column 3, are entered in column 5 . These values are plotted, on an appropriately chosen scale, against corresponding $P$-values, and a smooth curve that best fits the points is drawn in by inspection (residual plot, fig. 5). One may now locate the points, shown as circles, on this curve, whose abscissas are those observed for the chosen reference points. The corresponding ordinates are read off and inserted in the $\left(0.7 P+A_{R}\right)$ column. These values are marked with an asterisk for distinction. The corresponding values of $0.7 P$ are subtracted from $\left(A_{R}+0.7 P\right)$, and the resultant $A_{R}$ values, shown also with asterisks, are inserted in the vacant places of the $A_{R}$ column. These are the desired orders of interference at the several reference points. Values for $X_{R}(X=B, C, D$, ...) are obtained from all photographs in this manner.

${ }_{4}^{4}$ H. M. Goodwin, Elements of the precision of measurements and graphical methods, p. 60 (McGraw-Hill Book Co., New York, N. Y., 1920).
TABLE 2. Computation sheet for statistical evaluation of data The average of corresponding values in columns 3 and 5 represents the most acceptable results

\begin{tabular}{|c|c|c|c|c|}
\hline$R$ & $A_{R}$ & $Y_{R}$ & $B_{R}$ & $Y_{-R}$ \\
\hline-4 & & & & \\
\hline-3 & 6.80 & - & $\ldots$ & -....... \\
\hline-2 & $\begin{array}{l}5.50 \\
4.42\end{array}$ & $\ldots$ & --.-- & - \\
\hline $\begin{array}{r}-1 \\
0\end{array}$ & $\begin{array}{l}4.46 \\
3.33\end{array}$ & 0.00 & 8.71 & 0.00 \\
\hline+1 & 2.41 & -.09 & 7.13 & -.09 \\
\hline & 1. 62 & -.23 & 5. 75 & -.27 \\
\hline 3 & 0.98 & -.56 & 4. 49 & -.56 \\
\hline 4 & .39 & -.98 & 3. 32 & -1.04 \\
\hline 5 & - & -1.57 & 2.34 & -1.58 \\
\hline 6 & -..... & -2.30 & 1. 49 & -2.28 \\
\hline 7 & ....... & -3.16 & 0.77 & -3.13 \\
\hline 8 & & -4.26 & .29 & -4.20 \\
\hline 9 & ...... & -5.67 & & -5.39 \\
\hline 10 & ..... & -7.05 & - & -6.74 \\
\hline 11 & -...- & -8.64 & - & -8.28 \\
\hline 12 & -..-- & -10.30 & - & -9.96 \\
\hline 13 & - - & -12.37 & -..-.- & -11.86 \\
\hline 14 & -...- & -14.51 & ----. & -13.93 \\
\hline 15 & - & -16.86 & - & -16.21 \\
\hline
\end{tabular}

In order to determine the shape of the surface from the fringe readings and their corresponding abscissas, or $R$-values, these quantities are now applied to the formulas of eq (3), (4), and (6). Table 2 is a typical computation sheet. From eq (6) one computes $Y_{4}=3.33-\frac{1}{2}(8.23+0.39)=-0.98$, and likewise for all values of $Y_{R}$ in the range covered by the standard in position $A$ (column 3 of table 2 ). In order to proceed to the evaluation of $Y_{R}$ for other values of $R$, eq (3) and (4) are used. Values for $B_{R}$, found in the same manner as described previously for $A_{R}$, are inserted in column 4 , table 2 . This permits a summation to be made of all terms shown in eq (3) and (4) and consequently an evaluation of $M_{B}$ and $B_{0}$. As the number of reference points covered by the standard in each of the two positions, $A$ and $B$, is odd (i. e., from $R=0$ through $R=4$, or 5 points), they are each assumed to represent 2 observations, making the total number of observations 10 , which may now be divided into 2 equal groups. With this in view, it will be seen that $\sum_{R_{1}}^{R_{2}} R=2 \times 0+2 \times 1+1 \times 2=4$, and likewise for all summations shown in eq (3) and (4). The resultant values for $M_{B}$ and $B_{0}$ are, respectively, -1.580 and +8.67 . Slide-rule computation is adequate except for final precision. In general, the computed parameter $B_{0}$ will differ slightly from the observed value found in table 2. On substituting these values for $M_{X}$ and $X_{0}$ in eq (1), and using values for $R$ and $R_{R}$ in columns 1 and 4 , values for $Y_{R}$ may be computed from $R=5$ through 8 . These operations are now repeated, using consecutively the $C$ and $\mathrm{D}$ data, to compute additional values for $Y_{R}$ to the edge of the surface. These values are inserted in column 3, table 2. A similar treatment of the primed data yields an independent set of value for $Y_{-R}$ shown in column 5 , table 2 , which should agree approximately with the above set. An average of $Y_{R}$ and $Y_{-R}$ values for each value of $R$ is the accepted value. This averaging operation tends to decrease the effects of error in choice of the axis of rotation of the unknown surface as well as observational errors.

Washington, December 3, 1953. 